Editorial

\title{
Emerging Issues and Methodologies for Resilient and Robust Water Distribution Systems
}

\author{
Donghwi Jung $\mathbb{B}^{-1}$ and Joong Hoon Kim *๑ \\ School of Civil, Environmental and Architectural Engineering, Korea University, Anam-ro 145, Seongbuk-gu, \\ Seoul 02841, Korea; donghwiku@gmail.com \\ * Correspondence: jaykim@korea.ac.kr; Tel.: +82-2-3290-3316
}

Received: 5 March 2020; Accepted: 9 March 2020; Published: 11 March 2020

check for updates

\begin{abstract}
This editorial summarizes the 11 papers published in the Special Issue entitled "Resilient and Robust Water Distribution Systems: State-of-the-Art and Research Challenges" which were classified into five themes related to water distribution systems (WDSs): (1) state-of-the-art review on WDS resilience and robustness (ROB), (2) WDS performance quantification and recovery under earthquakes, (3) criticality analysis and visualization, (4) novel design methodologies, and (5) hydraulic parameter monitoring for WDS rapidity improvement. Following the provision of the number of views and citations of each paper in a brief manner, a paper in category (1) that reviewed recent studies on WDS robustness is summarized. Category (2) covers three papers on improving the WDS capacity to fulfil customers' demands in the case of an earthquake, a representative catastrophic failure event, while category (3) includes papers on visualization methods to represent the system's criticality. The studies included in themes (4) and (5) proposed novel design methods and monitoring approaches for improving WDS resilience, respectively. Contributions from each study are described in the context of WDS resilience. We hope that this Special Issue can (1) serve as a reference point from which readers review progress, recent trends, and emerging issues, and (2) shed light on the appropriate future directions of WDS resilience studies.
\end{abstract}

Keywords: resilience; robustness; water distribution system; design; operation and management

\section{Introduction}

The world is currently in an era of potential catastrophic disasters caused by climate change, e.g., mega-droughts, flash floods and earthquakes. Such globally climatic and environmental changes place severe stresses on water infrastructures, i.e., water distribution system (WDS) and urban drainage system (UDS). Earthquakes damage pipes and pumps in WDS, resulting in long-term service interruptions. Imbalances in water availability due to droughts instigate the construction of emergency pipelines.

The emergence of the concept of resilience may be taken for granted in the aforementioned uncertain and unprecedented circumstances. Resilience is introduced as a term referring to the scenario of humans effectively adapting to a natural system that does not behave as it did before and whose behavior cannot be anticipated based on historical observations. Some readers would consider "resilience" as another performance measure (compared to reliability and availability) that used to be a "hot topic" of focus, but this is not the case any longer. WDS resilience is defined as the system's ability to prepare, respond to and recover from catastrophic failure events. However, our WDS research domain lacks consistent efforts in terms of in-depth investigations and an understanding of each element of resilience, e.g., robustness (ROB), redundancy (RED), rapidity (RAP), and resourcefulness (RES). 
This Special Issue was prepared to facilitate the sharing of information on emerging issues and novel methodologies in the context of resilient and robust WDS. The originally intended scope of the Special Issue was as follows.

- $\quad$ Resilience-based WDS optimal design, operation, and management models

- Interdependence between ROB, RED, RAP, and RES (the four Rs of resilience)

- Smart metering for WDS rapidity and real-time operation and management (O\&M)

- High-performance optimization and machine learning algorithms

- WDS response and recovery under catastrophic failure events

- Anomaly detection, classification and location (e.g., pipe burst and leakage, cyber-attack, and intentional contamination)

- Water distribution network topology and resilience

- State-of-the-art review of WDS resilience metrics

- Defining emerging WDS resilience issues and problems

A total of 11 papers were published in the Special Issue and can be classified into five categories: (1) state-of-the-art review on WDS resilience and ROB (one paper); (2) WDS performance quantification and recovery methods during pre- and post-earthquake conditions (three papers); (3) novel (economic) criticality and failure analysis schemes and visualization approaches (two papers); (4) new design approaches for considering WDS redundancy, spatially variant demand patterns and segment isolation conditions (three papers); and, finally, (5) hydraulic parameter monitoring methodologies for WDS rapidity improvement (two papers). Therefore, interesting studies related to most of the intended themes were sought and included in the Special Issue; however, it could be confirmed that few efforts are currently being devoted to investigating the interdependency between the components of resilience, high-performance optimization and machine learning algorithms.

\section{Statistics of the Special Issue Contributions}

Table 1 summarizes the cumulative full-text and abstract views and number of citations in Google Scholar and Scopus of the papers published in the Special Issue. The daily average numbers of views are also included in parentheses in Table 1 . The papers are sorted in the order of publication date (Jun and Kwon [1], Yoo et al. [2], Choi and Kim [3], Diao et al. [4], Lee at al. [5], Jung et al. [6], Lee et al. [7], Kim et al. [8], Balut et al. [9], Lee et al. [10], Li et al. [11]).

Table 1. Metrics of papers published in this Special Issue (cumulative full-text and abstract views were calculated until 12 January 2019).

\begin{tabular}{|c|c|c|c|c|c|c|}
\hline \multirow{2}{*}{ Paper Reference } & \multirow{2}{*}{ Category ID } & \multirow{2}{*}{ Publication Date } & \multirow{2}{*}{$\begin{array}{c}\text { Full-Text Views } \\
\text { (Daily Avg) }\end{array}$} & \multirow{2}{*}{$\begin{array}{l}\text { Abstract Views } \\
\text { (Daily Avg) }\end{array}$} & \multicolumn{2}{|c|}{ Citations } \\
\hline & & & & & Google Scholar & Scopus \\
\hline Jun and Kwon [1] & 5 & 12 February 2019 & $629(2)$ & $516(2)$ & 0 & 0 \\
\hline Yoo et al. [2] & 2 & 15 February 2019 & $835(3)$ & $640(2)$ & 1 & 1 \\
\hline Choi and Kim [3] & 4 & 17 March 2019 & $573(2)$ & $561(2)$ & 2 & 2 \\
\hline Diao et al. [4] & 4 & 19 March 2019 & $589(2)$ & $748(3)$ & 0 & 0 \\
\hline Lee at al. [5] & 5 & 4 April 2019 & $500(2)$ & $659(2)$ & 1 & 0 \\
\hline Jung et al. [6] & 1 & 9 May 2019 & $815(3)$ & $580(2)$ & 1 & 1 \\
\hline Lee et al. [7] & 3 & 12 June 2019 & $533(2)$ & $568(3)$ & 1 & 1 \\
\hline Kim et al. [8] & 4 & 23 July 2019 & $647(4)$ & $756(4)$ & 0 & 0 \\
\hline Balut et al. [9] & 2 & 31 July 2019 & $443(3)$ & $544(3)$ & 1 & 0 \\
\hline Lee et al. [10] & 3 & 18 August 2019 & $526(4)$ & $619(4)$ & 1 & 0 \\
\hline Li et al. [11] & 2 & 30 November 2019 & $177(4)$ & $371(9)$ & 0 & 0 \\
\hline
\end{tabular}

Among the 11 papers, Yoo et al. [2] received the largest number of full-text views, whereas the abstract of Kim et al. [8] was viewed the most often. The average daily views of the papers tended to increase for papers published towards the due date of the Special Issue because the daily number of views tended to decrease over time. On the contrary, Choi and Kim [3] were found to be cited the most 
often (Google Scholar and Scopus). More citations of the 11 papers are expected as related follow-up studies are published in near future.

The papers in this Special Issue deal with five topics related to WDS resilience and ROB and are reviewed in the following sections:

- $\quad$ Section 3: State-of-the-Art Review on WDS Resilience and ROB

- Section 4: WDS Performance Quantification and Recovery under Earthquakes

- Section 5: Criticality Analysis and Visualization

- Section 6: Novel Design Methodologies

- Section 7: Hydraulic Parameter Monitoring for WDS Rapidity Improvement

\section{State-of-the-Art Review on WDS Resilience and ROB}

In the WDS domain, Lansey [12] was the first to comprehensively introduce and summarize the definition and examples of sustainability, resilience, and ROB in the context of WDS and its design, planning, and operation and management $(\mathrm{O} \& \mathrm{M})$. It should be noted that resilience and $\mathrm{ROB}$ were used as surrogate terminology and measures of reliability before Lansey's clarification [13]. Contributions from previous related works were also reviewed and discussed, while potential future problems with the metrics ideas and how to solve them were provided. These issues, with their methodologies, included how to cope in a water-scarce environment, the issues and gaps to first tackle in a new sensing paradigm (e.g., advanced metering infrastructure), and the manner by which to improve the effectiveness and efficiency of fault detection and water quality control.

Jung et al. [6] reviewed and summarized journal papers published in the context of WDS ROB that covered aspects Lansey [12] had been discussing since 2012. The state-of-the-art review of ROB measures and approaches was conducted in three research areas: design and planning, operation, and management. While most contributions proposed new ROB indicators for WDS design, pioneering works and efforts are being devoted to developing robust planning approaches for regional-scale water and waste infrastructures with high levels of uncertainty [14-16].

Jung et al. [6] confirmed that, when compared to the sequential ROB design studies, individual studies have been performed on WDS O\&M. Most O\&M studies proposed a methodology to limit the severity of the WDS performance in regard to optimal pump design and scheduling and valve installation. For example, Choi et al. [17] developed a so-called ROB-based valve installation method to minimize the maximum amount of undelivered segment demand under the case of a segment's failure for the recovery of a failed pipe. The state-of-the-art review paper on ROB was finalized with some future research topics: more efforts should be devoted to (1) the investigation of the interdependency among the four Rs of resilience: ROB, RED, RAP, and RES, and (2) the introduction of resilience and ROB concepts to various O\&M problems (e.g., development of operational unsteady ROB measures and the implementation of the ROB concept in the evaluation of sensing networks and systems).

\section{WDS Performance Quantification and Recovery under Earthquakes}

One of the features of WDS resilience that differentiates it from other traditional performance characteristics, such as reliability and availability, is the consideration of the system's multi-dimensional performance characteristics under "catastrophic failure events" [12], and not just a normal-abnormal event (e.g., a single pipe break). Earthquakes are one of the most representative natural disaster events that result in catastrophic failure consequences to WDS (e.g., multiple pipe breaks and power outages of pump stations), primarily because of a severe ground shift. Balut et al. [9], Li et al. [11] and Yoo et al. [2] contributed to WDS performance quantification and recovery under seismic events. Note that a pressure-driven analysis (PDA) was performed for a realistic network simulation under abnormal conditions in the three papers [2,9,11], although only Yoo et al. [2] used a full PDA approach that solved pressure-dependent nodal demands, pipe flows and nodal pressures simultaneously via a modified gradient method. 
Balut et al. [9] developed a pipe repair scheduling method based on a multi-criteria decisionmaking approach, Preference Ranking Organization METHod for Enrichment of Evaluations (PROMETHEE), for solving the Battle of Post-Disaster Response and Restoration problem at the 1st International Water Distribution Systems Analysis (WDSA)/International Computing \& Control for the Water Industry (CCWI) Joint Conference in 23-25 July 2018, in Kingston, Ontario, Canada. The prioritization of repairing or replacing multiple failed pipes caused by an earthquake was addressed in their study.

Two other studies focused on developing methodologies to evaluate the WDS performance during or after an earthquake event. Li et al. [11] introduced a seismic reliability quantification method considering two different types of acute stress, pipe leakage and breakage and fire flow simultaneously in an earthquake simulation. Yoo et al. [2] proposed a revised Reliability EVAluation model for seismic hazard for water supply NETwork (REVAS.NET) model [18] to implement PDA capability for a realistic leakage and pipe breakage simulation. In addition, a new surrogate measure of the WDS performance under earthquakes, the leakage ratio index, was developed to quantify the ratio of the amount of the total system leakage to the required total system demand.

\section{Criticality Analysis and Visualization}

A criticality analysis is one of the risk management approaches to identify failure modes (events) and potential losses in a system, which allows decision makers to rank and prioritize the modes [19]. Therefore, criticality analysis is performed to take pre-failure measures to supplement and improve the performance of the current system during future failure events. Hwang et al. [19] developed a failure mode effects and criticality analysis (FMECA) method for a regional water supply system based on the volumetric severity of the water demand quantified by a linear programming simulation model under various component failure conditions (e.g., pump station, water treatment plant, reservoir).

Two contributions by Lee et al. $[7,10]$ in this Special Issue were made in the context of WDS criticality analysis. Lee et al. [7] introduced a criticality analysis approach based on the modern portfolio theory (MPT), considering two different resilience measures, i.e., hydraulic and economic consequence resiliencies (HR and ER, respectively), to demonstrate the requirement of the economic measure in identifying critical pipes. Economic consequence resilience is defined as the ratio of delivered water to the required water weighted by economic consequences and output per unit of water under service interruptions. The proposed method was demonstrated in a hypothetical network in a U city where potential types of civil infrastructure service failures were pre-defined for each node, including broadcasting internet, telecommunication, hospital, and education services. They confirmed via a correlation analysis and comparison that there exists a minor association between the two resilience measures HR and ER, and the MPT with economic resilience tends to rank pipes with high economic consequences highly.

Lee et al. [10] proposed a set of visualization methods to display the relationship among failure mode, impact, and duration (or failure cause, impact, and duration (CID)). Two types of two-dimensional plots proposed in [10], the failure CID and CID bubble plots, were verified with 331 WDS component failure data points collected in South Korea between 1980 and 2018. They confirmed that the CID bubble plot is useful for identifying the most critical components (i.e., raw water pumping facilities, water treatment plants, water supply pipes and main distribution pipes).

\section{Novel Design Methodologies}

Two pre-failure system resilience characteristics, ROB and RED, can be secured through structural measures such as design and planning. The construction of a robust and redundant water network would result in a lower failure severity and functional degradation during failure events. Novel design methodologies for a resilient WDS were proposed in the following three publications in this Special Issue. 
Choi and Kim [3] investigated a trade-off relationship between the total system cost and mechanical redundancy (a kind of availability metric) of optimal design and layout solutions for WDS, which was performed under pipe break conditions with different degrees of failure (i.e., number of failed pipes is equal to $1,2, \ldots, 10)$. They observed that total system cost, mechanical reliability index, minimum nodal pressure, and system performance under fire flow conditions (from a post-optimization analysis) tended to converge and be consistent under the failure conditions with more than two pipe breaks. Because water network redundancy refers to the network's ability to secure alternative water supply paths to customer nodes, solving pipe size and layout (route) while simultaneously considering the water availability metric has about the same effect as considering network redundancy in the resulting designs.

Diao et al. [4] explored the impact of a spatially variant design demand pattern on the WDS design and operation. They compared the least costly design solutions obtained with uniform and spatially distributed demands with respect to the resulting capital cost, water age and pump operation cost. It was confirmed that, in the study networks considered, the latter case reduces capital cost by approximately $4.4 \%$, whereas water age is prolonged and both designs incur nearly equivalent pump operation costs. The authors finalized the paper by highlighting the need to investigate the temporal variability of design demands in addition to its spatial variability. Design demand is an input to the design model and problems and serves as a kind of stress to the system. Therefore, determining the characteristics and pattern is a critical task to construct a resilient WDS.

Kim et al. [8] proposed a heuristic algorithm to improve WDS reliability (the expected number of customers not receiving the service), quantified based on segment failure. The proposed approach determines the type of improvements among the cases of taking no action, increasing pipe size (and durability), and installing additional valves to the current network configuration. The expected magnitude of service interruptions was considered as a surrogate measure of failure severity and ROB. This study proved that the installation and operation of valves should be considered in WDS resilience studies that limit the service area affected by pipe failure.

\section{Hydraulic Parameter Monitoring for WDS Rapidity Improvement}

WDS rapidity is the system's ability to promptly detect anomalies (e.g., leakage, pipe break, cyberattack, water quality issue) and rapidly react and revert to its normal condition [12]. Therefore, implementing efficient early warning systems, maintaining the high preparedness of emergency recovery teams and constructing reliable and rapid two-way communication systems between the supervisory control and data acquisition system (SCADA) and remotely located booster stations could help improve the rapidity [20]. Contributions have been made regarding this topic by Jun and Kwon [1] and Lee et al. [5] who developed methods for pressure monitoring and chlorine injection locations, respectively.

Jun and Kwon [1] compared two pressure sensitivity analysis methods ("pressure contribution" and "pressure sensitivity" analyses) for locating pressure sensors which were verified by an unsteady transient pressure analysis based on the method of characteristics. To determine the pressure monitoring locations, the nodal pressure gradient (computed by the pressure change $(\Delta p)$ due to leakage divided by the base pressure value) was compared and ranked in the sensitivity analysis method, whereas the average oscillation height of the pressure transient was compared for different leakage locations in the unsteady method. Comparisons performed in a pilot plant network, a small example network and, finally, a real WDS in a H city confirmed that the proposed pressure sensitivity-based methods yield locations for pressure sensors similar to those obtained by the pressure transient method. The accurate and effective detection of leakages is the first step towards decreasing the average detection time (DT) (thus, effectiveness affects efficiency in this case), because failure to detect also implies an infinitely prolonged DT $(\mathrm{DT}=\infty)$, which significantly lowers the WDS rapidity [21].

Lee et al. [5] proposed an optimal chlorine injection (location and dosage) model to minimize the total mass of chlorine injected per day given the constraints on the residual chlorine concentration 
and the number of rechlorination facilities to be installed. Optimal solutions were sought by a greedy algorithm with a demand-driven analysis (DDA) and independent PDA for hydraulic and water quality simulations of a network for the constraint check. It was confirmed that a PDA-based solution tends to locate the injection points at an upstream divergence, whereas DDA-based solutions locate them at the end of a branched network. Similar to Yoo et al. [2], Lee et al. [5] highlighted the importance of a realistic network simulation (PDA) in performing WDS resilience studies that are mostly based on abnormal failure conditions (e.g., low pressure from a pipe break). In future studies, methodologies to minimize the risk originating from having multiple distributed rechlorination facilities (e.g., rechlorination ROB) could be developed to maintain the residual chlorine concentration, even under the failure of a single chlorine injection point.

\section{Conclusions}

This editorial summarized the 11 papers published in the Special Issue entitled "Resilient and Robust Water Distribution Systems: State-of-the-Art and Research Challenges" which were classified into 5 themes: (1) state-of-the-art review on WDS resilience and ROB; (2) WDS performance quantification and recovery under earthquakes; (3) criticality analysis and visualization; (4) novel design methodologies; (5) hydraulic parameter monitoring for WDS rapidity improvement. After briefly providing the number of views and citations of each paper, a paper in category (1) that reviewed recent studies for WDS robustness was summarized. While category (3) included visualization methods to represent the system's criticality, category (2) summarized three papers to improve the capacity of WDS to supply customers' demands under earthquakes, a representative catastrophic failure event for resilience. Studies included in themes (4) and (5) proposed novel design methods and monitoring approaches for improving WDS resilience, respectively. The contributions of each study were described in the context of WDS resilience.

Although the studies included in this Special Issue have made some contributions toward resilient WDS, there are research gaps that have not yet been explored. Among others, few efforts have been devoted to investigating the interdependence between the four subcomponents of WDS resilience: ROB, RED, RAP, and RES. In addition, the role of smart metering (e.g., advanced metering infrastructure) should be questioned and identified in terms of WDS resilience improvement. Finally, high-performance optimization and machine learning algorithms are essential for solving the aforementioned WDS resilience problems with real-world failure scenarios and conditions. In a follow-up Special Issue, we expect papers on the state-of-the-art review of WDS resilience metrics (not only ROB, but also RED, RAP, and RES) along with studies that address the aforementioned emerging WDS resilience issues and problems.

We hope this Special Issue can (1) serve as a reference point from which readers review progress, recent trends and emerging issues, and (2) shed light on the appropriate future directions of WDS resilience studies.

Author Contributions: Two authors, J.H.K. and D.J., have equivalently contributed to the literature review and summary, manuscript writing and the recommendations of potential research topics. All authors have read and agreed to the published version of the manuscript.

Funding: This research was supported by a grant (2019-MOIS31-010) from the Fundamental Technology Development Program for Extreme Disaster Response funded by the Korean Ministry of Interior and Safety (MOIS).

Acknowledgments: The guest editors would like to thank all the authors who contributed to this special issue as well as anonymous reviewers who provided their valuable comments which improved the scientific quality of the papers.

Conflicts of Interest: The authors declare no conflict of interest.

\section{References}

1. Jun, S.; Kwon, H.J. The optimum monitoring location of pressure in water distribution system. Water 2019, 11, 307. [CrossRef] 
2. Yoo, D.G.; Lee, J.H.; Lee, B.O. Comparative study of hydraulic simulation techniques for water supply networks under earthquake hazard. Water 2019, 11, 333. [CrossRef]

3. Choi, Y.H.; Kim, J.H. Development of multi-objective optimal redundant design approach for multiple pipe failure in water distribution system. Water 2019, 11, 553. [CrossRef]

4. Diao, K.; Sitzenfrei, R.; Rauch, W. The impacts of spatially variable demand patterns on water distribution system design and operation. Water 2019, 11, 567. [CrossRef]

5. Lee, S.M.; Lee, H.M.; Yoo, D.G.; Kim, J.H. A comparative study on a hydraulic and water-quality analysis method for determining rechlorination injection points for a water-supply network. Water 2019, 11, 697. [CrossRef]

6. Jung, D.; Lee, S.; Kim, J.H. Robustness and water distribution system: State-of-the-art review. Water 2019, 11, 974. [CrossRef]

7. Lee, S.; Shin, S.; Judi, D.R.; McPherson, T.; Burian, S.J. Criticality analysis of a water distribution system considering both economic consequences and hydraulic loss using modern portfolio theory. Water 2019, 11, 1222. [CrossRef]

8. Kim, S.; Jun, H.D.; Yoo, D.G.; Kim, J.H. A framework for improving reliability of water distribution systems based on a segment-based minimum cut-set approach. Water 2019, 11, 1524. [CrossRef]

9. Bałut, A.; Brodziak, R.; Bylka, J.; Zakrzewski, P. Ranking approach to scheduling repairs of a water distribution system for the post-disaster response and restoration service. Water 2019, 11, 1591. [CrossRef]

10. Lee, S.; Oak, S.; Jung, D.; Jun, H. Development of failure cause-impact-duration (CID) plots for water supply and distribution system management. Water 2019, 11, 1719. [CrossRef]

11. Li, Y.; Gao, J.; Zhang, H.; Deng, L.; Xin, P. Reliability assessment model of water distribution networks against fire following earthquake (FFE). Water 2019, 11, 2536. [CrossRef]

12. Lansey, K. Sustainable, robust, resilient, water distribution systems. In Proceedings of the 14th Water Distribution Systems Analysis Conference, Adelaide, Australia, 24-27 September 2012; p. 1.

13. Jung, D.; Kang, D.; Kim, J.H.; Lansey, K. Robustness-based design of water distribution systems. J. Water Res. Plan. Man. 2014, 140, 04014033. [CrossRef]

14. Kang, D.; Lansey, K. Scenario-based robust optimization of regional water and wastewater infrastructure. J. Water Res. Plan. Man. 2012, 139, 325-338. [CrossRef]

15. Kang, D.; Lansey, K. Multiperiod planning of water supply infrastructure based on scenario analysis. J. Water Res. Plan. Man. 2012, 140, 40-54. [CrossRef]

16. Ngo, T.T.; Jung, D.; Kim, J.H. Robust urban drainage system: Development of a novel multiscenario-based design approach. J. Water Res. Plan. Man. 2019, 145, 04019027. [CrossRef]

17. Choi, Y.H.; Jung, D.; Jun, H.; Kim, J.H. Improving water distribution systems robustness through optimal valve installation. Water 2018, 10, 1223. [CrossRef]

18. Yoo, D.G.; Jung, D.; Kang, D.; Kim, J.H.; Lansey, K. Seismic hazard assessment model for urban water supply networks. J. Water Res. Plan. Man. 2015, 142, 04015055. [CrossRef]

19. Hwang, H.; Lansey, K.; Quintanar, D.R. Resilience-based failure mode effects and criticality analysis for regional water supply system. J. Hydroinform. 2014, 17, 193-210. [CrossRef]

20. Jung, D.; Kim, J.H. State estimation network design for water distribution systems. J. Water Res. Plan. Man. 2017, 144, 06017006. [CrossRef]

21. Jung, D.; Kang, D.; Liu, J.; Lansey, K. Improving the rapidity of responses to pipe burst in water distribution systems: A comparison of statistical process control methods. J. Hydroinform. 2015, 17, 307-328. [CrossRef]

(C) 2020 by the authors. Licensee MDPI, Basel, Switzerland. This article is an open access article distributed under the terms and conditions of the Creative Commons Attribution (CC BY) license (http://creativecommons.org/licenses/by/4.0/). 